\title{
Stationary and convergent strategies in Choquet games
}

\author{
by \\ François G. Dorais (Ann Arbor, MI) and
Carl Mummert (Huntington, WV)
}

\begin{abstract}
If NONEMPTY has a winning strategy against EMPTY in the Choquet game on a space, the space is said to be a Choquet space. Such a winning strategy allows NonEMPTY to consider the entire finite history of previous moves before making each new move; a stationary strategy only permits NONEMPTY to consider the previous move by EMPTY. We show that NONEMPTY has a stationary winning strategy for every second-countable $T_{1}$ Choquet space. More generally, Nonempty has a stationary winning strategy for any $T_{1}$ Choquet space with an open-finite basis.

We also study convergent strategies for the Choquet game, proving the following results. A $T_{1}$ space $X$ is the open continuous image of a complete metric space if and only if NonemPTY has a convergent winning strategy in the Choquet game on $X$. A $T_{1}$ space $X$ is the open continuous compact image of a metric space if and only if $X$ is metacompact and Nonempty has a stationary convergent strategy in the Choquet game on $X$. A $T_{1}$ space $X$ is the open continuous compact image of a complete metric space if and only if $X$ is metacompact and NonEMPTY has a stationary convergent winning strategy in the Choquet game on $X$.
\end{abstract}

1. Introduction. The Choquet game is a Gale-Stewart game, denoted $\mathrm{Ch}(X)$, associated to a topological space $X$. There are two players, EMPTY and Nonempty, who alternate turns for $\omega$ rounds. On round $i$, EmPTy moves first, choosing $x_{i} \in X$ and an open set $U_{i}$ such that $x_{i} \in U_{i}$ and, if $i \geq 1$, such that $U_{i} \subseteq V_{i-1}$. Then NONEMPTY responds with an open set $V_{i}$ such that $x_{i} \in V_{i} \subseteq U_{i}$. After all the rounds have been played, EMPTY wins if $\bigcap_{n<\omega} V_{n}=\emptyset$. Otherwise, NONEMPTY wins $\left({ }^{1}\right)$.

A winning strategy for NONEMPTY is a function $\mathfrak{S}$ which takes a partial play of the game, ending with a move by EMPTY, and returns an open set

2010 Mathematics Subject Classification: Primary 91A24, 54D20; Secondary 06B35. Key words and phrases: Choquet game.

$\left({ }^{1}\right)$ The names of the two players EMPTY and NONEMPTY vary widely in the literature. They are sometimes called 0 and 1 , or 1 and 2 , or $\beta$ and $\alpha$, respectively. The Choquet game described here is sometimes called the strong Choquet game. 
for NonemPTy to play, such that NonemPTy will win any play of the game that follows the strategy. If NONEMPTY has a winning strategy for $\mathrm{Ch}(X)$ then $X$ is said to be a Choquet space.

The Choquet game was originally applied by Choquet [Cho69] to characterize complete metrizability of metric spaces. It has since been used more broadly to characterize completeness in arbitrary spaces. Kechris Kec95 describes the Choquet game, and its applications to descriptive set theory, in detail.

We are interested in the existence of two special types of strategies for NONEMPTY: stationary strategies and convergent strategies.

Definition 1.1. A stationary strategy for NonemPty in the game $\mathrm{Ch}(X)$ is a strategy $\mathfrak{S}$ that treats each move as the first move. That is,

$$
\mathfrak{S}\left(\left\langle x_{j}, U_{j}\right\rangle_{j \leq i}\right)=\mathfrak{S}\left(\left\langle x_{i}, U_{i}\right\rangle\right)
$$

for every partial play $\left\langle x_{j}, U_{j}\right\rangle_{j \leq i}$ of EMPTY against $\mathfrak{S}\left({ }^{2}\right)$.

Definition 1.2. A convergent strategy for NonEMPTY in the game $\mathrm{Ch}(X)$ is a strategy $\mathfrak{S}$ such that, for any play $\left\langle x_{i}, U_{i}, V_{i}\right\rangle_{i<\omega}$ following $\mathfrak{S}$, the collection $\left\{V_{i}: i<\omega\right\}$ is a neighborhood basis for any point in $\bigcap_{i} V_{i}$.

We do not require a stationary strategy or a convergent strategy to be a winning strategy for NonEMPTY. It is not hard to see that any $T_{0}$ space $X$ in which NonemPTy has a convergent strategy in $\mathrm{Ch}(X)$ is a $T_{1}$ space; thus some of our results have equivalent restatements in which " $T_{1}$ " is replaced with " $T_{0}$ ".

Galvin and Telgársky GT86 studied stationary strategies for generalized Choquet games. These games, and the results of Galvin and Telgársky, are discussed in Section 2.

It is well known that if $X$ is a complete metric space then NonEMPTY has a convergent stationary winning strategy for $\mathrm{Ch}(X)$. This is already implicit in the results of Choquet Cho69, where only stationary strategies are considered. However, the class of spaces for which NonEMPTy has a stationary winning strategy has not been characterized, and the class of spaces for which NONEMPTY has a convergent strategy is also uncharacterized.

Martin Mar03 proved that NONEMPTY has a winning strategy for every space that is representable as the set of maximal points of a directedcomplete partial order (d.c.p.o.) with the Scott topology. Such a space is said to be domain representable and must always be $T_{1}$. Martin established special cases when NONEMPTY has a stationary winning strategy, and asked whether there is always a stationary winning strategy. Our first theorem

$\left({ }^{2}\right)$ Stationary strategies are also called positional or memoryless strategies, or tactics. 
establishes that NONEMPTY has a stationary winning strategy for every second-countable domain representable space.

TheOREM 1.3. If $X$ is a second-countable $T_{1}$ space and Nonempty has a winning strategy in $\mathrm{Ch}(X)$ then NONEMPTY has a stationary winning strategy in $\mathrm{Ch}(X)$.

Mummert and Stephan [MS08] showed that the second-countable $T_{1}$ Choquet spaces are precisely the second-countable domain representable spaces, and these have an equivalent characterization in terms of representability by spaces of maximal filters called MF spaces. Combining this result with Martin's result and Theorem 1.3 gives the following corollary.

Corollary 1.4. Let $X$ be a second-countable $T_{1}$ space. Then Nonempty has a stationary winning strategy in $\mathrm{Ch}(X)$ if and only if $X$ is domain representable (equivalently, if and only if $X$ is representable as an MF space).

Bennett, Lutzer, and Reed [BLR08] established that a broad class of $T_{3}$ Choquet spaces admit stationary winning strategies for NonEMPTY. They asked [BLR08, Question 5.2] if there is an example of a $T_{3}$ domain representable space such that NONEMPTY does not have a stationary winning strategy. Corollary 1.4 shows that such an example, if it exists, cannot be second-countable.

The results in this paper do not assume separation beyond the $T_{1}$ axiom. Thus the results here are not a consequence of those of Bennett et al., because there are second-countable Hausdorff Choquet spaces that are nonmetrizable and thus not $T_{3}$. One example is the Gandy-Harrington space. The standard proof that the Gandy-Harrington space is a Choquet space, as presented by Kechris [Kec95], does not produce a stationary strategy for Nonempty, but Theorem 1.3 implies that there is such a strategy.

In Section 3, we study convergent strategies for NonEMPTY in the Choquet game. We obtain several theorems:

- (Theorem 3.3 A $T_{1}$ space $X$ is the open continuous image of a complete metric space if and only if NONEMPTY has a convergent winning strategy in $\mathrm{Ch}(X)$.

- (Theorem 3.8 A $T_{1}$ space $X$ is the open continuous compact image of a metric space if and only if $X$ is metacompact and NonEMPTY has a stationary convergent strategy in $\mathrm{Ch}(X)$.

- (Theorem 3.10 A $T_{1}$ space $X$ is the open continuous compact image of a complete metric space if and only if $X$ is metacompact and NONEMPTY has a stationary convergent winning strategy in $\mathrm{Ch}(X)$.

Here, a space $X$ is an open continuous compact image of a space $Y$ if there is an open continuous surjection $f: Y \rightarrow X$ such that $f^{-1}(\{x\})$ is compact for each $x \in X$. 
In light of these results, it is natural to ask whether a space $X$ is the open continuous image of a metric space if and only if NONEMPTY has a convergent strategy in $\mathrm{Ch}(X)$. In Example 3.5, we show that there is a first-countable $T_{1}$ space $X$ such that NonEMPTY does not have a convergent strategy in $\mathrm{Ch}(X)$. As Ponomarev Pon60 proved that every first-countable $T_{0}$ space is the open continuous image of a metric space, this example resolves the question with a negative answer.

Special bases. Our techniques for constructing convergent and stationary strategies require that the space being considered has bases with specific order properties. The study of such order properties is well established in the literature.

Definition 1.5. Let $\mathcal{B}$ be a basis for a topological space $X$ and, for every $x \in X$, let $\mathcal{B}_{x}=\{U \in \mathcal{B}: x \in U\}$ be the induced neighborhood basis at $x$. Then:

- $\mathcal{B}$ is Noetherian [LN76] if it satisfies the ascending chain condition. That is, every nondecreasing sequence of basis elements is eventually constant.

- $\mathcal{B}$ is open-finite $[$ Per76] if $\mathcal{B}[\supseteq U]$ is finite for every $U \in \mathcal{B}$. In other words, each set in the basis has only finitely many supersets in the basis.

- $\mathcal{B}$ is uniform [Ale60] if $\mathcal{B}_{x}[\nsubseteq U]$ is finite for every basic neighborhood pair $x \in U \in \mathcal{B}$. In other words, every infinite subset of $\mathcal{B}_{x}$ is a neighborhood basis at $x$.

- $\mathcal{B}$ is of countable order Arh63] if $\mathcal{B}_{x}[\not \amalg U]$ satisfies the descending chain condition for every basic neighborhood pair $x \in U \in \mathcal{B}$. In other words, every infinite descending chain in $\mathcal{B}_{x}$ is a neighborhood basis at $x$.

It follows immediately from definitions that every uniform basis is openfinite, and every open-finite basis is Noetherian. Moreover, every uniform basis is of countable order.

Some of our results make use of the following classical theorems, which indicate the utility of uniform bases. Lindgren and Nyikos [LN76] attribute the first of these to Aleksandrov.

THEOREM 1.6 (Aleksandrov Ale60]). A space has a uniform basis if and only if it is developable and metacompact.

Theorem 1.7 (Arhangel'skiu Arh62). Let $X$ be a $T_{1}$ space. Then $X$ has a uniform basis if and only if $X$ is the open continuous compact image of a metric space.

The previous two theorems show that the existence of a uniform basis cannot be demonstrated for general second-countable spaces, as there are 
nonmetacompact second-countable spaces, which can even be completely Hausdorff [SS78, \#69]. Our motivation for studying open-finite bases is that these can be obtained in very general circumstances.

Proposition 1.8. Every second-countable $T_{1}$ space has a countable openfinite basis.

Proof. We may assume that the space $X$ is not discrete (otherwise the result is trivial). Let $\left\langle U_{i}\right\rangle_{i<\omega}$ enumerate a basis of $X$, without repetitions, such that for every $i<\omega$, either $\left|U_{i}\right| \geq \omega$ or $\left|U_{i}\right|=1$.

Inductively choose $\left\langle x_{i}, V_{i}\right\rangle_{i<\omega}$ in such a way that if $U_{i}$ is a singleton then $V_{i}=U_{i}=\left\{x_{i}\right\}$, and if $\left|U_{i}\right|$ is infinite then $x_{i} \in V_{i}=U_{i} \backslash\left\{x_{j}: j<i\right\}$. Thus $i<j$ implies $x_{i} \in V_{i} \backslash V_{j}$ except when $V_{j}=\left\{x_{i}\right\}$. Therefore, each $V_{i}$ has at most $i+1$ supersets in $\mathcal{V}=\left\{V_{i}: i<\omega\right\}$.

If all elements of the sequence $\left\langle x_{i}\right\rangle_{i<\omega}$ are isolated points of $X$, then $\mathcal{V}$ itself is the required basis for $X$. Otherwise, let $\left\langle w_{k}\right\rangle_{k<\omega}$ enumerate the nonisolated points of $X$ that occur in the sequence $\left\langle x_{i}\right\rangle_{i<\omega}$, each with infinitely many repetitions. Then define

$$
W_{k}=\left(\bigcap\left\{U_{i}: i \leq k \wedge w_{k} \in U_{i}\right\}\right) \backslash\left\{x_{i}: i \leq k \wedge x_{i} \neq w_{k}\right\} .
$$

We claim that $\mathcal{B}=\mathcal{V} \cup \mathcal{W}$ is the required basis of $X$.

We first check that $\mathcal{B}$ is indeed a basis of $X$. It is enough to verify that if $x \in U_{i}$ then there is a $B \in \mathcal{B}$ with $x \in B \subseteq U_{i}$. If $x$ is an isolated point of $X$ or $x$ does not occur in $\left\langle x_{i}\right\rangle_{i<\omega}$, then $x \in V_{i} \subseteq U_{i}$. Otherwise, $x \in W_{k} \subseteq U_{i}$ where $k \geq i$ is such that $x=w_{k}$.

Next, we check that every $B \in \mathcal{B}$ has finitely many supersets in $\mathcal{B}$.

CASE $B=V_{i}$. We have already verified that every $V_{i}$ has finitely many supersets in $\mathcal{V}$. To see that $V_{i}$ has finitely many supersets in $\mathcal{W}$, note that if $k \geq i$, then either $w_{k} \neq x_{i}$ and $x_{i} \in V_{i} \backslash W_{k}$ (hence $V_{i} \nsubseteq W_{k}$ ), or $w_{k}=x_{i}$ and $W_{k} \subseteq V_{i}$ (hence $V_{i} \subseteq W_{k}$ implies $V_{i}=W_{k}$ ). Therefore $V_{i}$ has at most $i+1$ supersets in $\mathcal{W}$.

CASE $B=W_{k}$. Let $x_{i}=w_{k}$. If $j \geq \max (i, k)$ then either $w_{j} \neq w_{k}$ and $w_{k} \in W_{k} \backslash W_{j}$, or $w_{j}=w_{k}$ and $W_{j} \subseteq W_{k}$. Therefore there are at most $\max (i, k)+1$ elements of $\mathcal{W}$ that contain $W_{k}$. Also, if $j>i$ then $x_{i} \in W_{k} \backslash V_{j}$ so there are at most $i+1$ elements of $\mathcal{V}$ that contain $W_{k}$. Therefore, $W_{k}$ has finitely many supersets in $\mathcal{B}$.

2. Generalized Choquet games. Generalized Choquet games on a topological space $X$ are played exactly like the usual Choquet game on $X$, so that a play of the game determines a descending sequence $\left\langle U_{i}, V_{i}\right\rangle_{i<\omega}$ of open sets. The only difference lies in the way the winner is determined. We will be interested in games where NONEMPTY wins when the sequence $\left\langle U_{i}, V_{i}\right\rangle_{i<\omega}$ 
falls into some fixed payoff set of descending sequences of open sets. Thus, for example, the original Choquet game is defined with the payoff set containing all plays $\left\langle U_{i}, V_{i}\right\rangle_{i<\omega}$ such that $\bigcap_{i} V_{i}$ is nonempty. The generalized Choquet game associated with payoff set $P$ is denoted $\mathrm{Ch}_{P}(X)$. We will often think of the payoff set as defining a property shared by the winning plays of the game.

Although many instances of generalized Choquet games can be found in the literature, Galvin and Telgársky [GT86] were the first to explicitly consider this family of games. There is not much that one can say about $\mathrm{Ch}_{P}(X)$ for arbitrary $P$, since these are as general as Gale-Stewart games with arbitrary payoff sets. Thus our results will focus on classes of properties that are well-behaved.

DeFinition 2.1. Let $\left\langle U_{i}\right\rangle_{i<\omega}$ and $\left\langle V_{i}\right\rangle_{i<\omega}$ be descending sequences of open sets of a space $X$. We write $\left\langle U_{i}\right\rangle_{i<\omega} \leq\left\langle V_{i}\right\rangle_{i<\omega}$ if for each $V_{j}$ there is some $U_{i}$ with $U_{i} \subseteq V_{j}$. We write $\left\langle U_{i}\right\rangle_{i<\omega} \equiv\left\langle V_{i}\right\rangle_{i<\omega}$ if $\left\langle U_{i}\right\rangle_{i<\omega} \leq\left\langle V_{i}\right\rangle_{i<\omega}$ and $\left\langle V_{i}\right\rangle_{i<\omega} \leq\left\langle U_{i}\right\rangle_{i<\omega}$.

It is immediate that $\leq$ is a reflexive transitive relation and that $\equiv$ is an equivalence relation.

Definition 2.2. Let $P$ be a set of descending sequences of open sets of a space $X$. Then $P$ is:

- monotone if $\left\langle V_{i}\right\rangle_{i<\omega} \in P$ and $\left\langle U_{i}\right\rangle_{i<\omega} \leq\left\langle V_{i}\right\rangle_{i<\omega}$ implies $\left\langle U_{i}\right\rangle_{i<\omega} \in P$;

- invariant if $\left\langle V_{i}\right\rangle_{i<\omega} \in P$ and $\left\langle U_{i}\right\rangle_{i<\omega} \equiv\left\langle V_{i}\right\rangle_{i<\omega}$ implies $\left\langle U_{i}\right\rangle_{i<\omega} \in P$.

Every monotone property is invariant, but not conversely. In any play $\left\langle x_{i}, U_{i}, V_{i}\right\rangle_{i<\omega}$ of a generalized Choquet game, we have $\left\langle V_{i}\right\rangle_{i<\omega} \equiv\left\langle U_{i}\right\rangle_{i<\omega} \equiv$ $\left\langle U_{i}, V_{i}\right\rangle_{i<\omega}$, so for invariant properties it makes no difference which of these three sequences is tested to determine the outcome of the play.

Galvin and Telgársky considered monotone properties, obtaining the following general result.

TheOREm 2.3 (Galvin-Telgársky [GT86]). Let $P$ be a monotone property of descending sequences of open subsets of $X$. If NONEMPTY has a winning strategy in $\mathrm{Ch}_{P}(X)$ then NONEMPTY has a stationary winning strategy in $\mathrm{Ch}_{P}(X)$.

Unfortunately, the methods of Galvin and Telgársky rely heavily on monotonicity. A key example of an invariant property that is not monotone is the property " $\bigcap_{i} V_{i}$ is not empty" that defines the original Choquet game. In particular, Theorem 2.3 cannot be applied to Choquet games in the original sense.

In the following sections, we show that if $X$ has an open-finite basis and $P$ is an invariant property such that NONEMPTY has a winning strategy in 
$\mathrm{Ch}_{P}(X)$ then NonEmPTy has a stationary winning strategy in $\mathrm{Ch}_{P}(X)$. We also show that, for an invariant property $P$ on any space $X$, if NONEMPTY has a winning strategy in $\mathrm{Ch}_{P}(X)$ then NONEMPTY has a winning strategy in $\mathrm{Ch}_{P}(X)$ that only needs to remember the last point played by EMPTY and all of the previously played open sets, and thus does not need to know the other points played by EMPTY.

2.1. Basic properties. The invariant properties of descending sequences of open subsets of a space $X$ form a complete Boolean algebra of sets, as they are closed under arbitrary unions, arbitrary intersections, and complements. The monotone properties are similarly closed under arbitrary unions and intersections, but not under complements; so the monotone properties form a complete lattice of sets.

In this section, we study the subsets of these lattices consisting of the properties for which NONEMPTY has a winning strategy and the properties for which NONEMPTY has a stationary winning strategy. The next proposition shows that the properties $P$ for which NonEMPTy has a winning strategy in $\mathrm{Ch}_{P}(X)$ are closed under countable intersections in the algebra of invariant properties.

Proposition 2.4. Let $\langle P(k)\rangle_{k<\omega}$ be a sequence of invariant properties on a space $X$ and let $P=\bigcap_{k<\omega} P(k)$. If NonemPTy has a winning strategy in $\mathrm{Ch}_{P(k)}(X)$ for each $k<\omega$, then NONEMPTY has a winning strategy in $\mathrm{Ch}_{P}(X)$.

Proof. For each $k<\omega$, let $\mathfrak{S}_{k}$ be a winning strategy for NonEMPTY in $\mathrm{Ch}_{P_{k}}(X)$. Let $\langle\cdot, \cdot\rangle$ be a fixed bijection from $\omega \times \omega$ to $\omega$. We define the strategy $\mathfrak{S}$ by induction as follows. Given a partial play $\left\langle x_{i}, U_{i}, V_{i}\right\rangle_{i \leq n}$ against $\mathfrak{S}$, write $n=\langle k, m\rangle$ and then define

$$
\mathfrak{S}\left\langle x_{i}, U_{i}\right\rangle=\mathfrak{S}_{k}\left\langle x_{\langle k, j\rangle}, U_{\langle k, j\rangle}\right\rangle_{j \leq m} .
$$

For any play $\left\langle x_{i}, U_{i}, V_{i}\right\rangle_{i<\omega}$ against $\mathfrak{S}$, the sequence $\left\langle x_{\langle k, j\rangle}, U_{\langle k, j\rangle}, V_{\langle k, j\rangle}\right\rangle_{j<\omega}$ is a play against $\mathfrak{S}_{k}$. Since $\left\langle V_{i}\right\rangle_{i<\omega} \equiv\left\langle V_{\langle k, j\rangle}\right\rangle_{j<\omega}$, we see that $\left\langle V_{i}\right\rangle_{i<\omega} \in P(k)$. Since this is true for every $k<\omega$, we conclude that $\left\langle V_{i}\right\rangle_{i<\omega} \in P$. Therefore, $\mathfrak{S}$ is a winning strategy for NONEMPTY in $\mathrm{Ch}_{P}(X)$.

It follows from Theorem 2.3 that the monotone properties $P$ for which Nonempty has a stationary winning strategy in $\mathrm{Ch}_{P}(X)$ form a filter which is closed under countable intersections in the lattice of monotone properties. The set of invariant properties $P$ for which NONEMPTy has a stationary winning strategy in $\mathrm{Ch}_{P}(X)$ forms a filter in the algebra of invariant properties.

Proposition 2.5. Let $P_{1}$ and $P_{2}$ be invariant properties. If Nonempty has winning stationary strategies in $\mathrm{Ch}_{P_{2}}(X)$ and $\mathrm{Ch}_{P_{2}}(X)$, then NONEMPTY has a stationary winning strategy in $\mathrm{Ch}_{P_{1} \cap P_{2}}(X)$. 
Proof. Given winning stationary strategies $\mathfrak{S}_{1}$ and $\mathfrak{S}_{2}$ for NonEMPTY in $\mathrm{Ch}_{P_{1}}(X)$ and $\mathrm{Ch}_{P_{2}}(X)$, respectively, define $\mathfrak{S}(x, U)=\mathfrak{S}_{2}\left(x, \mathfrak{S}_{1}(x, U)\right)$. If $\left\langle x_{i}, U_{i}, V_{i}\right\rangle_{i<\omega}$ is a play of EMPTY against $\mathfrak{S}$ and $W_{i}=\mathfrak{S}_{1}\left(x_{i}, U_{i}\right)$ for each $i<\omega$, then $\left\langle x_{i}, W_{i}, V_{i}\right\rangle_{i<\omega}$ is a play against $\mathfrak{S}_{2}$ and $\left\langle x_{i}, U_{i}, W_{i}\right\rangle_{i<\omega}$ is a play against $\mathfrak{S}_{1}$. Thus $\left\langle V_{i}\right\rangle_{i<\omega} \in P_{2}$ and $\left\langle W_{i}\right\rangle_{i<\omega} \in P_{1}$, but since $\left\langle V_{i}\right\rangle_{i<\omega} \equiv$ $\left\langle W_{i}\right\rangle_{i<\omega}$, it follows that $\left\langle V_{i}\right\rangle_{i<\omega} \in P_{1}$ as well.

When $X$ has an open-finite basis, Theorem 2.16 below and Proposition 2.4 can be employed to show that the filter of invariant properties for which NONEMPTY has a stationary winning strategy is closed under countable intersections. We do not have a full characterization of the spaces with this property.

QUESTION 2.6. For what spaces $X$ is the filter of invariant properties $P$ for which NONEMPTY has a stationary winning strategy in $\mathrm{Ch}_{P}(X)$ closed under countable intersections?

From time to time, we will find it useful to restrict the moves of the players to some fixed basis $\mathcal{B}$ for the space $X$. For any property $P$ of descending sequences of open sets, we let $\mathrm{Ch}_{P}(X, \mathcal{B})$ refer to the variant of the generalized Choquet game on $X$ in which both players are constrained to play open sets from the basis $\mathcal{B}$. Strategies for this game are similarly restricted. Such restrictions have no impact on the determinacy of games in which the defining property is invariant.

Proposition 2.7. Let $\mathcal{B}$ be a basis for the space $X$ and let $B$ be a function such that, for every neighborhood pair $x \in U$, we have $x \in B(x, U) \subseteq U$ and $B(x, U) \in \mathcal{B}$. If $P$ is an invariant property, then:

(i) If $\mathfrak{S}$ is a winning strategy for NONEMPTY in $\mathrm{Ch}_{P}(X, \mathcal{B})$ then

$$
\mathfrak{S}^{\prime}\left(\left\langle x_{i}, U_{i}\right\rangle_{i \leq n}\right)=\mathfrak{S}\left(\left\langle x_{i}, B\left(x_{i}, U_{i}\right)\right\rangle_{i \leq n}\right)
$$

defines a winning strategy for NONEMPTY in $\mathrm{Ch}_{P}(X)$.

(ii) If $\mathfrak{S}$ is a winning strategy for NONEMPTY in $\mathrm{Ch}_{P}(X)$ then

$$
\mathfrak{S}^{\prime \prime}\left(\left\langle x_{i}, U_{i}\right\rangle_{i \leq n}\right)=B\left(x_{n}, \mathfrak{S}\left(\left\langle x_{i}, U_{i}\right\rangle_{i \leq n}\right)\right)
$$

defines a winning strategy for NONEMPTY in $\mathrm{Ch}_{P}(X, \mathcal{B})$.

In each case, if the original strategy was stationary then so is the modified strategy.

Proof. (i) Fix a play $\left\langle x_{n}, U_{n}, V_{n}\right\rangle_{n<\omega}$ of $\mathrm{Ch}_{P}(X)$ in which NonemPty follows $\mathfrak{S}^{\prime}$. By definition of $\mathfrak{S}^{\prime}$, we always have $V_{n}=\mathfrak{S}\left\langle x_{i}, \widehat{U}_{i}\right\rangle_{i \leq n}$ where $\widehat{U}_{i}=B\left(x_{i}, U_{i}\right)$. Thus $\left\langle x_{n}, \widehat{U}_{n}, V_{n}\right\rangle$ is a play of $\mathrm{Ch}_{P}(X, \mathcal{B})$ wherein NONEMPTY used $\mathfrak{S}$. Since $\mathfrak{S}$ is winning for NonemPTy, we have $\left\langle V_{n}\right\rangle_{n<\omega} \in P$.

(ii) Fix a play $\left\langle x_{n}, U_{n}, V_{n}\right\rangle_{n<\omega}$ of $\mathrm{Ch}_{P}(X, \mathcal{B})$ in which NonEmPTy used $\mathfrak{S}^{\prime \prime}$. By definition of $\mathfrak{S}^{\prime \prime}$, we always have $V_{n}=B\left(x_{n}, \widehat{V}_{n}\right)$ where $\widehat{V}_{n}=$ 
$\mathfrak{S}\left\langle x_{i}, U_{i}\right\rangle_{i \leq n}$. Thus $\left\langle x_{n}, U_{n}, \widehat{V}_{n}\right\rangle_{n<\omega}$ is a play of $\operatorname{Ch}_{P}(X)$ wherein NonEmPty used $\mathfrak{S}$. Since $\mathfrak{S}$ is winning for NonemPTY, we have $\left\langle\widehat{V}_{n}\right\rangle_{n<\omega} \in P$. Moreover, since

$$
\widehat{V}_{n+1} \subseteq U_{n+1} \subseteq V_{n} \subseteq \widehat{V}_{n},
$$

we have $\left\langle V_{n}\right\rangle_{n<\omega} \equiv\left\langle\widehat{V}_{n}\right\rangle_{n<\omega}$, which means that $\left\langle V_{n}\right\rangle_{n<\omega} \in P$, by the invariance of $P$.

A similar result holds for winning strategies for EMPTY, but we will have no use for that result, because we only study winning strategies for NONEMPTY.

2.2. Trace strategies. Trace strategies force Nonempty to ignore all points played by EMPTY except the most recent point, and thus only consider the sequence of open sets that have been played before the latest move. This is a much weaker restriction on a strategy than stationarity, allowing trace strategies to be obtained in more general circumstances. We will show that a winning trace strategy can always be found when NONEMPTY has a winning strategy in $\mathrm{Ch}_{P}(X)$, without further assumptions on $X$. In Section 2.3, trace strategies serve as an intermediate step in the path towards stationary strategies. In Section 3 , they are used to improve cardinality results in circumstances when we cannot obtain stationary strategies.

Definition 2.8. The open trace of a (possibly partial) play $\left\langle x_{i}, U_{i}, V_{i}\right\rangle_{i<n}$ of $\mathrm{Ch}_{P}(X)$ is the set $\left\{\left\langle U_{i}, V_{i}\right\rangle: i<n\right\}$ of pairs of open sets that have been played by the two players.

Definition 2.9. A trace strategy for Nonempty in $\mathrm{Ch}_{P}(X)$ is a strategy $\mathfrak{S}$ where each move for NONEMPTY depends only on EMPTY's last move and the open trace of the previous moves. In other words, there is a function $\mathfrak{S}^{*}$ such that

$$
\mathfrak{S}\left(\left\langle x_{i}, U_{i}\right\rangle_{i \leq n}\right)=\mathfrak{S}^{*}\left(x_{n}, U_{n},\left\{\left\langle U_{i}, V_{i}\right\rangle: i<n\right\}\right),
$$

where as usual $V_{i}=\mathfrak{S}\left(\left\langle x_{j}, U_{j}\right\rangle_{j \leq i}\right)$ for $i<n$.

Before proving the existence of trace strategies, we need to eliminate some strange behavior that is admissible in general strategies for GaleStewart games but serves no purpose in generalized Choquet games with invariant payoff sets. One reason behind the definition of an open trace as the set of previous moves, rather than the sequence of previous moves, is to reduce difficulties caused by possibility that the players play the same move repeatedly. However, additional work is required to completely remove the effects of repetition from arbitrary strategies.

Definition 2.10. A strategy $\mathfrak{S}$ for Nonempty in a generalized Choquet game is stable if $\mathfrak{S}\left(x_{0}, U_{0} ; \ldots ; x_{n}, U_{n}\right)=U_{n}$ implies that $\mathfrak{S}\left(x_{0}, U_{0} ; \ldots ; x_{n}, U_{n} ; x_{n}, U_{n} ; \ldots ; x_{m}, U_{m}\right)=\mathfrak{S}\left(x_{0}, U_{0} ; \ldots ; x_{n}, U_{n} ; \ldots ; x_{m}, U_{m}\right)$. 
In other words, NONEMPTY's responses are unaffected if EMPTY repeats the same move two (or more) times in a play.

Every trace strategy is stable; every winning strategy for an invariant property can be made into a stable strategy.

Proposition 2.11. Let $P$ be an invariant property and let $\mathcal{B}$ be an arbitrary basis for $X$. If NONEMPTY has a winning strategy in $\mathrm{Ch}_{P}(X)$ then NONEMPTY has a stable winning strategy in $\mathrm{Ch}_{P}(X, \mathcal{B})$.

Proof. By Proposition 2.7, we may assume that we have a winning strategy $\mathfrak{S}$ for Nonempty in $\mathrm{Ch}_{P}(X, \mathcal{B})$. We will refine Nonempty's original strategy $\mathfrak{S}$ in two phases.

We first define the strategy $\mathfrak{S}^{\prime}$ to follow $\mathfrak{S}$ in all cases except when $\mathfrak{S}\left(\left\langle x_{i}, U_{i}\right\rangle_{i \leq n}\right)=U_{n}$. When this happens, we scan ahead repeating EMPTY's last move until S's response is different from $U_{n}$. If this never happens, we set $\mathfrak{S}^{\prime}\left(\left\langle x_{i}, U_{i}\right\rangle_{i \leq n}\right)=U_{n}$. If, after some number $r$ of repetitions, we get a different answer $U_{n}^{\prime}$, we define $\mathfrak{S}^{\prime}\left(\left\langle x_{i}, U_{i}\right\rangle_{i \leq n}\right)=U_{n}^{\prime}$ and pretend that EMPTY's $n$th move was repeated $r$ times in all future queries to $\mathfrak{S}$. Since $P$ is invariant, the play without repetitions is winning for NONEMPTY if and only if the play with repetitions is winning for NONEMPTY.

Next we define $\mathfrak{S}^{\prime \prime}$ from $\mathfrak{S}^{\prime}$ as follows. Whenever EMPTY repeats a move, NONEMPTY initially responds (as $\mathfrak{S}^{\prime}$ requires) with EMPTY's last played open set. However, if EMPTY suddenly plays differently, NONEMPTY collapses EMPTY's repeated plays to a single play before querying $\mathfrak{S}^{\prime}$ on this and all future rounds. Again, since $P$ is invariant, the play with repetitions is winning for NONEMPTY if and only if the play without repetitions is winning for NonEmPTY. The strategy $\mathfrak{S}^{\prime \prime}$ is a stable winning strategy for NONEMPTy in $\mathrm{Ch}_{P}(X)$.

Our next theorem implies the existence of winning trace strategies for NonemPTY in every Choquet space.

THEOREM 2.12. Let $P$ be an invariant property and let $\mathcal{B}$ be an arbitrary basis for $X$. If NONEMPTY has a winning strategy in $\mathrm{Ch}_{P}(X)$ then NONEMPTY has a winning trace strategy in $\mathrm{Ch}_{P}(X, \mathcal{B})$.

Proof. Let $\mathfrak{S}$ be a stable winning strategy for NonEMPTY in $\mathrm{Ch}_{P}(X, \mathcal{B})$ as per Proposition 2.11. (This is the only place where we use the fact that $P$ is invariant.) We will use $\mathfrak{S}$ to construct a function $\mathfrak{S}^{*}$, which in turn defines a trace strategy $\mathfrak{S}_{t}$ (as above) with

$$
\mathfrak{S}_{t}\left(\left\langle x_{i}, U_{i}\right\rangle_{i \leq n}\right)=\mathfrak{S}^{*}\left(x_{n}, U_{n}, T_{n}\right),
$$

where

$$
T_{n}=\left\{\left\langle U_{i}, \mathfrak{S}_{t}\left(\left\langle x_{j}, U_{j}\right\rangle_{j \leq i}\right)\right\rangle: i<n\right\} .
$$

We then show that $\mathfrak{S}_{t}$ is a winning strategy for NonEMPTY in $\operatorname{Ch}_{P}(X, \mathcal{B})$. 
To ensure that $\mathfrak{S}_{t}$ is indeed a winning strategy for NoNEMPTY, we will simultaneously define an auxiliary function $w$ mapping nonempty open traces of partial plays to points in $X$. This function $w$ will have the property that if $\left\langle x_{i}, U_{i}, V_{i}\right\rangle_{i<\omega}$ is any play against $\mathfrak{S}_{t}$, then $\left\langle y_{i}, U_{i}, V_{i}\right\rangle_{i<\omega}$ is a play against $\mathfrak{S}$, where $y_{i}=w\left(\left\{\left\langle U_{j}, V_{j}\right\rangle: j \leq i\right\}\right)$ for $i<\omega$.

The definition of $\mathfrak{S}^{*}(x, U, T)$ proceeds by induction on $|T|$. As a base case, we define $\mathfrak{S}^{*}(x, U, \emptyset)=\mathfrak{S}(x, U)$; we do not need to define $w(\emptyset)$.

Suppose that we have specified $\mathfrak{S}^{*}(x, U, T)$ for all open traces of size less than $n$. Let $\left\langle x_{i}, U_{i}\right\rangle_{i \leq n}$ be a partial play for EMPTY against $\mathfrak{S}_{t}$, and let $T_{n}$ be the corresponding open trace. In order to determine $\mathfrak{S}_{t}\left(\left\langle x_{i}, U_{i}\right\rangle_{i \leq n}\right)$, we will define $V_{n}=\mathfrak{S}^{*}\left(x_{n}, U_{n}, T_{n}\right)$ and then define $y_{n}=w\left(T_{n+1}\right)$, where as above $T_{n+1}=T_{n} \cup\left\{\left\langle U_{n}, V_{n}\right\rangle\right\}$. For convenience, write $V_{i}=\mathfrak{S}_{t}\left(\left\langle x_{j}, U_{j}\right\rangle_{j \leq i}\right)$ for $i<n$. By the induction hypothesis, if we define $y_{i}=w\left(\left\{\left\langle U_{j}, V_{j}\right\rangle: j \leq i\right\}\right)$ for $i<n$, then we also have $V_{i}=\mathfrak{S}\left(\left\langle y_{j}, U_{j}\right\rangle_{j \leq i}\right)$ for every $i<n$. Moreover, we must choose $y_{n}$ and $V_{n}$ so that $V_{n}=\mathfrak{S}\left(\left\langle y_{i}, U_{i}\right\rangle_{i \leq n}\right)$ in order to preserve the induction hypothesis.

If $\left|T_{n}\right|<n$, then $V_{n}=\mathfrak{S}^{*}\left(x_{n}, U_{n}, T_{n}\right)$ and $y_{n}=w\left(T_{n}\right)$ have already been defined. However, the only way that $\left|T_{n}\right|<n$ could happen is if $\left\langle y_{i}, U_{i}\right\rangle_{i \leq n}$ includes redundant moves by EMPTY. Since $\mathfrak{S}$ is a stable strategy, we must have $V_{n}=\mathfrak{S}\left(\left\langle y_{i}, U_{i}\right\rangle_{i \leq n}\right)$, as this equality was satisfied for the play obtained by contracting all redundant moves from $\left\langle y_{i}, U_{i}\right\rangle_{i \leq n}$.

Now suppose $\left|T_{n}\right|=n$. Using Zorn's Lemma, find a maximal set $Y \subseteq U_{n}$ such that the map $y \mapsto V_{y}$ is an injection, where $V_{y}=\mathfrak{S}\left(\left\langle v_{i}, U_{i}\right\rangle_{i \leq n}\right), v_{i}=y_{i}$ for $i<n$, and $v_{n}=y$. We necessarily have $U_{n}=\bigcup_{y \in Y} V_{y}$. So we can pick $y_{n} \in Y$ and $V_{n}=V_{y_{n}}$ such that $x_{n} \in V_{n}$. The fact that $y \mapsto V_{y}$ is an injection guarantees that defining $w\left(T_{n+1}\right)=y_{n}$ is sound.

2.3. Stationary strategies. Before proving our main result on the existence of stationary winning strategies in generalized Choquet games on spaces with open-finite bases, we will give a general criterion for the existence of winning stationary strategies in generalized Choquet games on any space. The motivating idea is that a stationary strategy for NonEMPTY in the Choquet game should respond to a move $\langle x, U\rangle$ with a neighborhood of $x$ that is very small compared to $U$.

Definition 2.13. Let $X$ be a space with a basis $\mathcal{B}$, and let $\mathfrak{S}$ be a strategy for NonemPTy in $\mathrm{Ch}_{P}(X, \mathcal{B})$. We say that $\langle x, U, V\rangle$ is a good triple for $\mathfrak{S}$ if $U \in \mathcal{B}, V \in \mathcal{B}, x \in V \subseteq U$, and $V$ is contained in every response of $\mathfrak{S}$ to a partial play by EMPTY against $\mathfrak{S}$ ending with the move $\langle x, U\rangle$. That is, $\langle x, U, V\rangle$ is a good triple for $\mathfrak{S}$ if for every partial play $\left\langle x_{i}, U_{i}, V_{i}\right\rangle_{i<n}$ of $\mathrm{Ch}_{P}(X, \mathcal{B})$ against $\mathfrak{S}$ such that $U \subseteq \bigcap_{i<n} V_{i}$, we have

$$
V \subseteq \mathfrak{S}\left(x_{0}, U_{0} ; \ldots ; x_{n-1}, U_{n-1} ; x, U\right) .
$$


We often suppress $\mathfrak{S}$, and simply say that $\langle x, U, V\rangle$ is a good triple, when NonEMPTY's strategy is clear from context.

Definition 2.14. We say that $\mathfrak{S}$ has enough good triples, relative to a given basis $\mathcal{B}$, if for every open neighborhood $U \in \mathcal{B}$ of a point $x$ there is an open neighborhood $V \in \mathcal{B}$ of $x$ such that $\langle x, U, V\rangle$ is a good triple for $\mathfrak{S}$.

If $\mathfrak{S}$ is a stationary winning strategy, then $\langle x, U, V\rangle$ is a good triple if and only if $x \in V \subseteq \mathfrak{S}(x, U)$. Therefore, a stationary strategy always has enough good triples. On the other hand, any winning strategy for NONEMPTY with enough good triples leads to a stationary winning strategy for NONEMPTY.

Proposition 2.15. If $P$ is an invariant property, then Nonempty has a stationary winning strategy in $\mathrm{Ch}_{P}(X)$ if (and only if) there is a basis $\mathcal{B}$ for $X$ such that NonEMPTY has a winning strategy in $\mathrm{Ch}_{P}(X, \mathcal{B})$ with enough good triples.

Proof. Suppose that $\mathfrak{S}$ is a winning strategy for NonemPTY in $\mathrm{Ch}_{P}(X, \mathcal{B})$ that has enough good triples. Define a stationary strategy $\mathfrak{S}_{s}$ in $\mathrm{Ch}_{P}(X, \mathcal{B})$ by simply choosing, when presented with a move $\langle x, U\rangle$, some $V \in \mathcal{B}$ such that $\langle x, U, V\rangle$ is a good triple for $\mathfrak{S}$.

Let $\left\langle x_{i}, U_{i}, V_{i}\right\rangle_{i<\omega}$ be a play of $\operatorname{Ch}_{P}(X, \mathcal{B})$ following $\mathfrak{S}_{s}$. We must show that $\left\langle V_{i}\right\rangle_{i<\omega} \in P$. This is done by constructing a sequence $\left\langle V_{i}^{\prime}\right\rangle_{i<\omega}$ of elements of $\mathcal{B}$ such that $V_{i+1} \subseteq V_{i+1}^{\prime} \subseteq V_{i}$ for all $i \in \omega$ and such that $\left\langle x_{i}, U_{i}, V_{i}^{\prime}\right\rangle_{i<\omega}$ is a play of of $\operatorname{Ch}_{P}(X, \mathcal{B})$ following $\mathfrak{S}$. Since $\left\langle V_{i}\right\rangle_{i<\omega} \equiv\left\langle V_{i}^{\prime}\right\rangle_{i<\omega}$ and $P$ is invariant, it will immediately follow that $\left\langle V_{i}\right\rangle_{i<\omega} \in P$.

We proceed inductively. At round 0, EMPTY picks $\left\langle x_{0}, U_{0}\right\rangle$; so $V_{0}$ is chosen to complete a good triple. Define $V_{0}^{\prime}=\mathfrak{S}\left(x_{0}, U_{0}\right)$, which means $V_{0} \subseteq$ $V_{0}^{\prime}$ by definition of good triple.

Now at round $i+1$, we may assume by induction that $V_{i} \subseteq V_{i}^{\prime}$. EMPTY has played $x_{i+1} \in U_{i+1} \subseteq V_{i}$, which means that $\left\langle x_{i+1}, U_{i+1}\right\rangle$ would be a legal move for EMPTY in response to the partial play $\left\langle x_{k}, U_{k}, V_{k}^{\prime}\right\rangle_{k \leq i}$ in $\mathrm{Ch}_{P}(X, \mathcal{B})$. Now $\left\langle x_{i+1}, U_{i+1}, V_{i+1}\right\rangle$ is a good triple, so we know that $V_{i+1}^{\prime}=$ $\mathfrak{S}\left(\left\langle x_{k}, U_{k}\right\rangle_{k \leq i+1}\right)$ has the property that $x_{i+1} \in V_{i+1} \subseteq V_{i+1}^{\prime} \subseteq U_{i+1} \subseteq V_{i}$. Continuing this process through all $\omega$ rounds produces the desired play of $\mathrm{Ch}_{P}(X, \mathcal{B})$.

We are now prepared to prove our main result on the existence of stationary winning strategies in generalized Choquet games. This result applies, in particular, to the original Choquet game and to its variant in which NONEMPTY is additionally required to follow a convergent strategy.

Theorem 2.16. Let $X$ be a space with an open-finite basis $\mathcal{B}$ and let $P$ be an invariant property of descending sequences of open subsets of $X$. If Nonempty has a winning strategy in $\mathrm{Ch}_{P}(X)$ then Nonempty has a stationary winning strategy in $\mathrm{Ch}_{P}(X)$. 
Proof. We will show that every trace strategy for NonEMPTY in $\mathrm{Ch}_{P}(X, \mathcal{B})$ has enough good triples. This is sufficient, because if NONEMPTY has a winning strategy in $\mathrm{Ch}_{P}(X)$ then NONEMPTY has a winning trace strategy in $\mathrm{Ch}_{P}(X, \mathcal{B})$ by Theorem 2.12. If this trace strategy has enough good triples, then Nonempty has a stationary winning strategy in $\mathrm{Ch}_{P}(X)$ by Proposition 2.15 .

Let $\mathfrak{S}$ be a winning trace strategy for Nonempty in $\mathrm{Ch}_{P}(X, \mathcal{B})$. Let $\mathcal{T}_{U}$ be the set of all open traces of finite partial plays following $\mathfrak{S}$ for which $\langle x, U\rangle$ is a valid next move for EMPTY. Since $\mathcal{B}$ is open-finite, there are only finitely many pairs of open sets that can occur in elements of $\mathcal{T}_{U}$, because each set in the pair must be a superset of $U$. Therefore, $\mathcal{T}_{U}$ is finite, and hence the set

$$
W=\bigcap\left\{\mathfrak{S}^{*}(x, U, T): T \in \mathcal{T}_{U}\right\}
$$

is an open neighborhood of $x$. If $V \in \mathcal{B}$ is a neighborhood of $x$ with $V \subseteq W$ then $\langle x, U, V\rangle$ is a good triple for $\mathfrak{S}$.

Theorem 1.3 is an immediate consequence of Theorem 2.16 and Proposition 1.8 .

3. Convergent strategies. When $X$ is a metric space, either NoNEMPTY or EMPTY can ensure that the intersection of open sets in a play of the Choquet game consists of at most one point, by selecting open sets of smaller and smaller radius as the play progresses. We generalize this to nonmetric spaces via the notion of convergent strategies, as defined in the introduction. Thus, if $X$ is $T_{1}$, the intersection of open sets in a play of $\mathrm{Ch}(X)$ following a convergent strategy contains at most one point. However, we do not require a convergent strategy to be a winning strategy for NONEMPTY.

The work of Galvin and Telgársky GT86 can be directly applied to study convergent strategies, as the following proposition demonstrates.

Proposition 3.1. Nonempty has a convergent strategy in $\mathrm{Ch}(X)$ if and only if NONEMPTY has a stationary convergent strategy in $\mathrm{Ch}(X)$. (This strategy may not be a winning strategy in $\mathrm{Ch}(X)$.)

Proof. Define a property $P$ consisting of all descending sequences $\left\langle U_{i}\right\rangle_{i<\omega}$ of open sets of $X$ such that $\left\{U_{i}: i \in \omega\right\}$ is a neighborhood basis for every point in $\bigcap_{i<\omega} U_{i}$. To say that NonEMPTY has a convergent strategy for $\mathrm{Ch}(X)$ is exactly the same as saying that NonEMPTY has a winning strategy in $\mathrm{Ch}_{P}(X)$. Because $P$ is a monotone property, Theorem 2.3 applies to $\mathrm{Ch}_{P}(X)$, allowing any winning strategy in $\mathrm{Ch}_{P}(X)$ to be converted to a stationary winning strategy for $\mathrm{Ch}_{P}(X)$, which in turn is a stationary convergent stategy for $\mathrm{Ch}(X)$. 
The proposition that any open continuous image of a Choquet space is itself a Choquet space is listed as an exercise by Kechris [Kec95, 8.16]. We isolate the proof here so that we can refer to it during the proof of Theorem 3.3 .

Proposition 3.2. Assume that $\mathfrak{S}_{Z}$ is a winning strategy for NonEMPTY in $\mathrm{Ch}(Z)$ and that there is an open continuous surjection from $Z$ to $X$. Then there is a winning strategy $\mathfrak{S}_{X}$ for NONEMPTY in $\mathrm{Ch}(X)$.

Proof. Let $\mathfrak{S}_{Z}$ be a winning strategy for NonemPTy in $\mathrm{Ch}(Z)$ and let $f: Z \rightarrow X$ be an open continuous surjection. We inductively define a strategy $\mathfrak{S}_{X}$ for NonemPTy in $\mathrm{Ch}(X)$. The construction uses a back-and-forth technique following the diagram below:

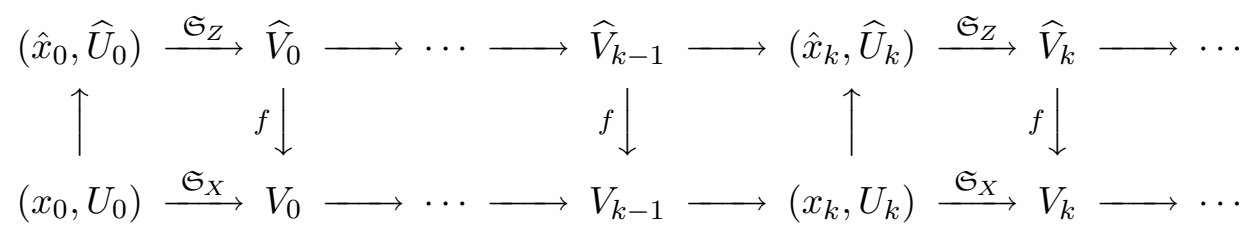

At round 0 , given $x_{0} \in U_{0} \subseteq X$, choose some $\hat{x}_{0} \in Z$ with $f\left(\hat{x}_{0}\right)=x_{0}$, and let $\widehat{U}_{0}=f^{-1}\left(U_{0}\right)$. Then $\hat{x}_{0} \in \widehat{U}_{0}$. Let $\widehat{V}_{0}=\mathfrak{S}_{Z}\left(\hat{x}_{0}, \widehat{U}_{0}\right) ;$ so $\hat{x}_{0} \in \widehat{V}_{0}$, which means $f\left(\hat{x}_{0}\right)=x_{0} \in f\left(\widehat{V}_{0}\right)$. Let $V_{0}$ be $f\left(\widehat{V}_{0}\right)$. Because $\widehat{V}_{0} \subseteq \widehat{U}_{0}=f^{-1}\left(U_{0}\right)$, we have $V_{0} \subseteq U_{0}$. Thus $V_{0}$ is a legal first move for NonEMPTY in response to $\left\langle x_{0}, U_{0}\right\rangle$.

Now at stage $k>0$, given $\left\langle x_{k}, U_{k}\right\rangle$, let $\widehat{U}_{k}=f^{-1}\left(U_{k}\right) \cap \widehat{V}_{k-1}$. We know that $x_{k} \in U_{k} \subseteq f\left(\widehat{V}_{k-1}\right)$, which means that there is some point $\hat{x}_{k} \in \widehat{V}_{k-1}$ with $f\left(\hat{x}_{k}\right)=x_{k}$. Because $f\left(\hat{x}_{k}\right)=x_{k} \in U_{k}$, we see that $\hat{x}_{k} \in f^{-1}\left(U_{k}\right)$, and thus $\hat{x}_{k} \in \widehat{U}_{k}$. Also, $\widehat{U}_{k} \subseteq \widehat{V}_{k-1}$, by construction, which means that $\left\langle\hat{x}_{k}, \widehat{U}_{k}\right\rangle$ is a legal move for EMPTY in $\mathrm{Ch}(Z)$ in response to the partial play $\left\langle\hat{x}_{0}, \widehat{U}_{0}, \ldots, \widehat{V}_{k-1}\right\rangle$. Let $\widehat{V}_{k}=\mathfrak{S}_{Z}\left(\hat{x}_{0}, \widehat{U}_{0}, \ldots, \hat{x}_{k}, \widehat{U}_{k}\right)$ and define $V_{k}=f\left(\widehat{V}_{k}\right)$. Because $\widehat{V}_{k} \subseteq \widehat{U}_{k} \subseteq f^{-1}\left(U_{k}\right)$, we have $V_{k} \subseteq U_{k}$. Because $\hat{x}_{k} \in \widehat{V}_{k}$, we have $x_{k} \in V_{k}$. Thus $V_{k}$ is a legal move for NonemPTY in $\mathrm{Ch}(X)$ for this round.

Our first theorem of this section characterizes the $T_{1}$ spaces for which there is a convergent strategy for NONEMPTY in the Choquet game.

Theorem 3.3. Let $X$ be a $T_{1}$ space. Then $X$ is the open continuous image of a complete metric space if and only if NONEMPTY has a convergent winning strategy in $\mathrm{Ch}(X)$. Moreover, the metric space can be taken to have the same weight as $X$.

Proof. For the forward direction, suppose $f: Z \rightarrow X$ is an open continuous surjection from a complete metric space $Z$ to a $T_{1}$ space $X$. Let $\mathfrak{S}_{Z}$ be a convergent winning trace strategy for NONEMPTY in $\mathrm{Ch}(Z)$ with the property that the open sets played by NONEMPTY in any play following $\mathfrak{S}_{Z}$ have 
radii converging to 0, and thus the sequence of points played by EMPTY is a Cauchy sequence. The canonical winning strategy for NonEMPTY in $\mathrm{Ch}(Z)$ has these properties. Construct a winning strategy $\mathfrak{S}_{X}$ for NONEMPTY in $\mathrm{Ch}(X)$ exactly as in the proof of Proposition 3.2 .

We must prove that $\mathfrak{S}_{X}$ is a convergent strategy. Let $\left\langle x_{k}, U_{k}\right\rangle_{k<\omega}$ be a play of $\operatorname{Ch}(X)$ following $\mathfrak{S}_{X}$. Then there is a corresponding play $\left\langle\hat{x}_{k}, \widehat{U}_{k}\right\rangle_{k<\omega}$ as defined in the construction of $\mathfrak{S}_{Z}$, and a corresponding sequence $\left\langle\widehat{V}_{k}\right\rangle_{k<\omega}$.

Because $\mathfrak{S}_{Z}$ is a convergent winning strategy for NonEMPTY in $\mathrm{Ch}(Z)$, there is a single point $z \in \bigcap_{i} \widehat{U}_{i}$, which is the limit of the sequence $\left\langle\hat{x}_{i}\right\rangle_{i<\omega}$. To see that $U=\bigcap_{i} U_{i}$ is a singleton, suppose $y$ and $z$ are distinct points of $U$. Then there is a sequence $\left\langle\hat{y}_{i}\right\rangle_{i<\omega}$ such that $\hat{y}_{i} \in \widehat{U}_{i}$ and $f\left(\hat{y}_{i}\right)=y$, and a sequence $\left\langle\hat{z}_{i}\right\rangle_{i<\omega}$ such that $\hat{z}_{i} \in \widehat{U}_{i}$ and $f\left(\hat{z}_{i}\right)=z$. Now, because the radii of $\left\langle\widehat{U}_{i}\right\rangle_{i<\omega}$ converge to 0 , and $Z$ is a complete space, both $\left\langle\hat{y}_{i}\right\rangle_{i<\omega}$ and $\left\langle\hat{z}_{i}\right\rangle_{i<\omega}$ are convergent, and have the same limit $\hat{l}$. Now let $W \subseteq X$ be an open neighborhood of $z$ with $y \notin W$. Then $\hat{l} \in f^{-1}(W)$ and so $\left\langle\hat{y}_{i}\right\rangle_{i<\omega}$ is eventually in $f^{-1}(W)$, which is impossible because $y \notin W$ but $f\left(\hat{y}_{i}\right)=y$.

This shows that $U$ contains a single point $z=f(\hat{z})$. Now let $W^{\prime} \subseteq X$ be any open neighborhood of $z$. Then $f^{-1}\left(W^{\prime}\right)$ is an open neighborhood of $\hat{z}$, and so $\widehat{V}_{k} \subseteq f^{-1}\left(W^{\prime}\right)$ for some $k$. Then $z \in V_{k} \subseteq W^{\prime}$; this shows that $\mathfrak{S}_{X}$ is a convergent winning strategy for NONEMPTY.

For the converse, assume that $X$ is a $T_{1}$ space and that Nonempty has a convergent winning strategy $\mathfrak{S}$ in $\mathrm{Ch}(X)$. Note that the property that a play of $\mathrm{Ch}(X)$ is convergent and winning is an invariant property. Thus, by Theorem 2.12, we may assume $\mathfrak{S}$ is a trace strategy.

Let $\mathcal{B}$ be any basis for $X$ and let $S \subseteq \prod_{n<\omega} \mathcal{B}^{2}$ be the set of all descending sequences $\left\langle U_{n}, V_{n}\right\rangle_{n<\omega}$ of pairs of elements of $\mathcal{B}$ such that for every $n<\omega$ there is some $x \in U_{n}$ such that

$$
U_{n+1} \subseteq V_{n}=\mathfrak{S}\left(U_{n}, x,\left\{\left\langle U_{i}, V_{i}\right\rangle: i<n\right\}\right) .
$$

Now $\prod_{n<\omega} \mathcal{B}^{2}$ has a natural complete metric: the distance between two sequences is $2^{-n}$ when $n$ is the index of the first position where the sequences differ. Moreover, $S$ is closed as a subset of $\prod_{n<\omega} \mathcal{B}^{2}$, and thus $S$ is a complete metric space.

Any sequence $\left\langle U_{n}, V_{n}\right\rangle_{n<\omega} \in S$ will have a single point of $X$ in $\bigcap_{i} V_{i}$, because $\mathfrak{S}$ is a convergent winning strategy for NonEMPTY and $X$ is $T_{1}$. Thus there is a well-defined map $f: S \rightarrow X$ such that $f\left(\left\langle U_{n}, V_{n}\right\rangle_{n<\omega}\right)$ is the unique element of $\bigcap_{n} V_{n}$. To check that this map is continuous, fix a point $x$ in an open set $U$, and a sequence $s \in S$ with $f(s)=x$. Then, because $\mathfrak{S}$ is convergent, there is some $n$ such that $s(n) \subseteq U$. The set of all sequences in $S$ that agree with $s$ on the first $n$ coordinates is an open neighborhood $\widehat{U}$ of $s$ with $f(\widehat{U}) \subseteq U$. 
To check that $f$ is an open mapping, let $U$ be a basic open set in $S$. Without loss of generality, $U$ is determined by a finite initial segment $\tau=$ $\left\langle U_{1}, V_{1}, \ldots, U_{n}, V_{n}\right\rangle$ of open sets. Now for any $x \in V_{n}$, there is an extension of $\tau$ to an element $s_{x}$ with $f\left(s_{x}\right)=x$, which is obtained by simply playing $x$ and a neighborhood basis of $x$ in the Choquet game. Thus $f(U)=V_{n}$.

Finally, we verify that the weight of the metric space can be taken to be the same as the weight of $X$. If $X$ is finite, then because $X$ is $T_{1}$ it is discrete, and the result is trivial. Now suppose that $X$ is infinite and $\mathcal{B}$ is a basis for $X$ of minimal cardinality; $\mathcal{B}$ will be infinite as well. Now the space $S$, if constructed from $\mathcal{B}$ as above, has a basis whose cardinality is no larger than the cardinality of the set of finite subsets of $\mathcal{B}^{2}$. This will be exactly the cardinality of $\mathcal{B}$.

Our next example shows that the completeness assumption in Theorem 3.3 cannot be removed altogether. We will rely on a characterization of the first-countable spaces due to Ponomarev.

Theorem 3.4 (Ponomarev [Pon60]). A $T_{0}$ space is first-countable if and only if it is the open continuous image of a metric space.

The space in the following example has been discussed by Todorčević Tod84.

EXAMPLE 3.5. There is a first-countable Hausdorff space $X$ such that there is no convergent strategy for NONEMPTY in $\mathrm{Ch}(X)$. Moreover, by Theorem 3.4, this space is the open continuous image of a metric space.

Proof. The example relies on several concepts from set theory that we define briefly here; these are not used outside of the present proof. The set of countable ordinals is denoted $\omega_{1}$. A subset of $\omega_{1}$ is unbounded if it has no upper bound less than $\omega_{1}$, and closed when it is closed in the order topology. A set is $c l u b$ if it is closed and unbounded, and stationary if it has nonempty intersection with every club set. Every club set is stationary, and it is well known that there are stationary sets that do not contain any club set. A function $f$ from an initial segment of $\omega_{1}$ to $\omega_{1}$ is continuous if it is continuous in the order topology.

Fix a set $A \subseteq \omega_{1}$ that is stationary and does not contain any club set; thus $\omega_{1} \backslash A$ is unbounded in $\omega_{1}$. We construct our example $X=X_{A}$ as the set of all maximal paths through a certain tree $T$. For each ordinal $\alpha<\omega_{1}$, let $T_{\alpha}$ consist of all continuous, increasing functions from the ordinals less than or equal to $\alpha$ to $\omega_{1} \backslash A$. Then let $T=\bigcup_{\alpha<\omega_{1}} T_{\alpha}$. We assign $X$ the topology in which each element $\tau$ of $T$ determines a basic open set $N_{\tau}$, consisting of those elements of $X$ that extend $\tau$.

Any maximal path $f$ through $T$ can be naturally identified with a continuous increasing function from an initial segment $\operatorname{dom}(f) \subseteq \omega_{1}$ to $\omega_{1} \backslash A$. 
Moreover, $o(f)=\sup \{f(\alpha): \alpha \in \operatorname{dom}(f)\}$ will be an element of $A$ or will be $\omega_{1}$. For, if $o(f)<\omega_{1}$ is not in $A$, then we could extend $f$ to a larger continuous increasing function, because we have assumed that $\omega_{1} \backslash A$ is unbounded.

For any $f \in X$, if $o(f)=\omega_{1}$, then $\operatorname{dom}(f)=\omega_{1}$ and $C=\left\{f(\alpha): \alpha<\omega_{1}\right\}$ will be a club set. In this case, because $A$ is a stationary set, $C \cap A$ is nonempty, contradicting the definition of $f$. Thus, each maximal path $f$ through $T$ has a bounded range, and thus a bounded domain, so there is some sequence $\langle\tau(i)\rangle_{i<\omega}$ with $f=\bigcap N_{\tau(i)}$. This means that $X$ is firstcountable.

Now suppose that $\mathfrak{S}$ is a convergent strategy for NonEMPTY in $\mathrm{Ch}(X)$; we will show that $A$ contains a club set, namely the set

$$
C=\{o(f): f \in T \text { and } o(f) \in A\} .
$$

As this set is clearly a subset of $A$, we only need to prove it is closed and unbounded. Note that, because a descending sequence of nonempty open sets of $X$ cannot have an empty intersection, $\mathfrak{S}$ will necessarily be a winning strategy. Moreover, because $X$ is Hausdorff, any play of $\operatorname{Ch}\left(X_{A}\right)$ that follows $\mathfrak{S}$ will have a single point in the intersection of the open sets played.

To see that $C$ is unbounded, note that for any $\beta<\omega_{1}$, the set of ordinals between $\beta$ and $\omega_{1}$ that are of the form $o(f)$ for some $f \in T$ will be a club set, which will have nonempty intersection with the stationary set $A$.

Let $\left\langle\alpha_{i}\right\rangle_{i<\omega}$ be any increasing sequence of elements of $C$. To complete the proof that $C$ is a club set, we must show that $\alpha=\sup \alpha_{i}$ is in $C$. We define a play of $\operatorname{Ch}(X)$ that follows $\mathfrak{S}$. At stage 0 , find some element $f_{0} \in X$ with $o\left(f_{0}\right)=\alpha_{0}$; this is possible because of the definition of $C$. Make EMPTY play $f_{0}$ and any open neighborhood of $f_{0}$, so that $\mathfrak{S}$ returns $V_{0}$. We may assume that $V_{0}$ is a basic open neighborhood, determined by a continuous increasing function $g_{0}$ from an initial segment of $\omega_{1}$ to $\omega_{1} \backslash A$. Now, because $f_{0} \in V_{0}$, there is no ordinal in the range of $g_{0}$ that is larger than $\alpha_{0}$. Thus, because $\operatorname{dom}\left(g_{0}\right)$ has a largest element and $\alpha_{0}$ is a limit ordinal, we can extend $g_{0}$ to some $f_{1} \in V_{0}$ such that $o\left(f_{1}\right)=\alpha_{1}$, and then find a basic neighborhood $U_{1}$ of $f_{1}$ such that every $f \in U_{1}$ has $o(f)>\alpha_{0}$. Let $V_{1}$ be the response of $\mathfrak{S}$ when EMPTY now plays $\left\langle f_{1}, U_{1}\right\rangle$; then $V_{1}$ is determined by some function $g_{1} \in T$. Continuing inductively, we generate a play $\left\langle f_{i}, U_{i}\right\rangle_{i<\omega}$ of $\mathrm{Ch}(X)$ following $\mathfrak{S}$, and a corresponding sequence $\left\langle g_{i}\right\rangle_{i<\omega}$.

Because $\mathfrak{S}$ is a convergent winning strategy, there is a unique point $f \in \bigcap_{i} U_{i}$. Moreover, $f=\bigcup_{i} g_{i}$, because otherwise there would be more than one point in $\bigcap_{i} U_{i}$. Now we have $\alpha=\sup \alpha_{i}=o(f)$, and we proved above that $o(f)$ must be in $A$. By the definition of $C$, this implies that $\alpha \in C$, which is what we wanted to prove. Thus, if NonEMPTy has a convergent strategy in $\operatorname{Ch}(X)$, then $A$ contains the club set $C$. This contradicts the 
assumption that $A$ is stationary but does not contain a club set; thus there is no convergent strategy for NONEMPTY in $\mathrm{Ch}(X)$.

Before we prove the remaining theorems from the introduction, we require two propositions about spaces with specific kinds of bases.

Proposition 3.6. If a $T_{1}$ space $X$ has a basis of countable order, then NONEMPTY has a stationary convergent strategy in $\mathrm{Ch}(X)$. (This strategy may not be a winning strategy.)

Proof. Suppose $\mathcal{B}$ is a basis of countable order for the space $X$. Define a stationary strategy $\mathfrak{S}$ by letting $V=\mathfrak{S}(x, U)$ be any element of $\mathcal{B}$ such that $x \in V \subsetneq U$, unless that is impossible, in which case $U=\mathfrak{S}(x, U)$. The second case can only occur if $U=\{x\}$.

Suppose that $\left\langle x_{i}, U_{i}\right\rangle_{i<\omega}$ is a play of $\mathrm{Ch}(X)$ following $\mathfrak{S}$. Suppose $x \in$ $\bigcap_{i} U_{i}$. If the set $\mathcal{U}=\left\{U_{i}: i<\omega\right\}$ is infinite, then $\mathcal{U}$ is a neighborhood basis for $x$ because $\mathcal{B}$ is of countable order. Otherwise, $\mathcal{U}$ is finite, in which case there is some $U \in \mathcal{U}$ which is a minimal open neighborhood for $x$; this also means that $\mathcal{U}$ is a neighborhood basis for $x$.

The proof of the following proposition is similar to the proof of Choquet's theorem presented by Kechris [Kec95, Sec. 8.E]

Proposition 3.7. A $T_{1}$ space $X$ has a uniform basis if and only if $X$ is metacompact and NONEMPTY has a stationary convergent strategy in $\mathrm{Ch}(X)$. (This strategy may not be a winning strategy.)

Proof. For the forward implication, suppose $\mathcal{B}$ is a uniform basis for $X$. We know from Theorem 1.6 that $X$ is metacompact. Also, $\mathcal{B}$ is of countable order, which implies that NONEMPTY has a stationary convergent strategy in $\mathrm{Ch}(X)$ by Proposition 3.6.

For the reverse implication, suppose that $X$ is metacompact and that $\mathfrak{S}$ is a stationary convergent strategy for NonEMPTY in $\mathrm{Ch}(X)$.

We use $\mathfrak{S}$ to inductively construct a uniform basis $\mathcal{B}$ for $X$ in $\omega$ stages. At stage 0 , set $\mathcal{B}_{0}=\{X\}$, which is a point-finite open cover of $X$. At stage $i+1$, having defined the point-finite open cover $\mathcal{B}_{i}$, pick $\mathcal{B}_{i+1}$ to be a point-finite open refinement of the family $\left\{\mathfrak{S}(x, U): x \in U \in \mathcal{B}_{i}\right\}$. We claim that $\mathcal{B}=\bigcup_{i<\omega} \mathcal{B}_{i}$ is a uniform basis for $X$.

To see this, suppose that $\mathcal{A} \subseteq \mathcal{B}$ is infinite and that $x \in \bigcap \mathcal{A}$. Let $\widehat{\mathcal{A}}$ be the upward closure of $\mathcal{A}$ in $\mathcal{B}$. Now $\widehat{\mathcal{A}}$ is a basis at $x$ only if $\mathcal{A}$ is a basis at $x$. Since $\mathcal{B}_{i}$ is point-finite and $x$ belongs to every element of $\widehat{\mathcal{A}}$, the set $\widehat{\mathcal{A}} \cap \mathcal{B}_{i}$ is finite for each $i<\omega$.

Consider the finitely branching tree $\mathcal{T}$ of finite sequences $\left\langle U_{i}\right\rangle_{i \leq n}$ such that $U_{i} \in \widehat{\mathcal{A}} \cap \mathcal{B}_{i}$ and, if $i \geq 1$, then $U_{i} \subseteq \mathfrak{S}\left(w, U_{i-1}\right)$ for some $w \in U_{i}$. 
The definition of $\left\langle\mathcal{B}_{i}\right\rangle_{i<\omega}$ guarantees that every element of $\widehat{\mathcal{A}}$ belongs to a sequence in $\mathcal{T}$. Since $\widehat{\mathcal{A}}$ is infinite it follows that $\mathcal{T}$ is also infinite.

By König's Lemma, $\mathcal{T}$ has an infinite branch $\left\langle U_{i}\right\rangle_{i<\omega}$. By definition of $\mathcal{T}$, we can pick a sequence of points $\left\langle w_{i}\right\rangle_{i<\omega}$ such that $\left\langle w_{i}, U_{i}\right\rangle_{i<\omega}$ is a play of the game $\operatorname{Ch}(X)$ following $\mathfrak{S}$. Since $\mathfrak{S}$ is convergent, it follows that $\left\{U_{i}\right\}_{i<\omega} \subseteq \widehat{\mathcal{A}}$ is a neighborhood basis at $x$, hence $\mathcal{A}$ is also a neighborhood basis at $x$.

The next theorem parallels Theorem 3.3 it characterizes the $T_{1}$ metacompact spaces for which NONEMPTY has a convergent strategy in the Choquet game.

Theorem 3.8. Let $X$ be a $T_{1}$ space. Then $X$ is the open continuous compact image of a metric space if and only if $X$ is metacompact and NONEMPTY has a convergent strategy in $\mathrm{Ch}(X)$. Moreover, if these conditions hold, then NONEMPTY has a stationary convergent strategy in $\mathrm{Ch}(X)$. (This strategy may not be a winning strategy.)

Proof. Let $X$ be a $T_{1}$ space. By Theorem 1.7, $X$ is the open continuous compact image of a metrizable space if and only if $X$ has a uniform basis. But, by Proposition 3.7, $X$ has a uniform basis if and only if $X$ is metacompact and NonemPTY has a stationary convergent strategy in $\mathrm{Ch}(X)$.

Our next theorem will require an additional result on metacompactness.

Proposition 3.9. Let $X$ and $Y$ be $T_{1}$ spaces and let $f$ be an open continuous compact mapping of $X$ onto $Y$. If $X$ is paracompact then $Y$ is metacompact.

Proof. Let $\mathcal{V}_{0}$ be an open cover of $Y$ and let $\mathcal{U}_{0}=\left\{f^{-1}(V): V \in \mathcal{V}_{0}\right\}$, which is an open cover of $X$. Since $X$ is paracompact, we can find a locallyfinite open refinement $\mathcal{U}$ of $\mathcal{U}_{0}$. We claim that $\mathcal{V}=\{f(U): U \in \mathcal{U}\}$ is a point-finite open refinement of $\mathcal{V}_{0}$.

The fact that $\mathcal{V}$ is an open refinement of $\mathcal{V}_{0}$ is clear. Since $f$ is open and onto, $\mathcal{V}$ is certainly an open cover of $Y$. For each $U \in \mathcal{U}$ there is a $V \in \mathcal{V}_{0}$ such that $U \subseteq f^{-1}(V)$. Therefore, $f(U) \subseteq V$, which shows that $\mathcal{V}$ is a refinement of $\mathcal{V}_{0}$.

It remains to show that $\mathcal{V}$ is point-finite. Fix $y \in Y$. For each $x \in X$, we can find an open neighborhood $W_{x}$ of $x$ that meets only finitely many elements of $\mathcal{U}$. Because $f$ is a compact mapping, $f^{-1}(y)$ is a compact subset of $X$. So we can find $x_{1}, \ldots, x_{k} \in f^{-1}(y)$ such that $f^{-1}(y) \subseteq W_{x_{1}} \cup \cdots \cup W_{x_{k}}$. If $U \in \mathcal{U}$ and $y \in f(U)$ then $U \cap W_{x_{i}} \neq \emptyset$ for some $i \in\{1, \ldots, k\}$. By our choice of $W_{x_{i}}$, there are only finitely many such $U \in \mathcal{U}$ for each $i \in$ $\{1, \ldots, k\}$. Therefore, the set $\{V \in \mathcal{V}: y \in V\}$ is finite.

Our final theorem gives a characterization of the $T_{1}$ metacompact spaces for which NonEmPTy has a convergent winning strategy for the Choquet game. 
Theorem 3.10. Let $X$ be a $T_{1}$ space. Then $X$ is the open continuous compact image of a complete metric space if and only if $X$ is metacompact and NonemPTY has a convergent winning strategy in $\mathrm{Ch}(X)$. Moreover, the strategy can be taken to be stationary, and the metric space can be taken to have the same weight as $X$.

Proof. First, assume that $f: Z \rightarrow X$ is an open continuous compact surjection from a complete metric space $Z$ to $X$. It follows from Theorem 3.3 that NonempTy has a convergent winning strategy in $\mathrm{Ch}(X)$, and it follows from Proposition 3.9 that $X$ is metacompact. The proof of the converse implication will show that we can take the strategy to be stationary.

For the converse, assume $X$ is a metacompact $T_{1}$ space and NonEMPTY has a convergent winning strategy for $\mathrm{Ch}(X)$. By Propositions 3.1 and 3.7 . there is a uniform basis $\mathcal{B}$ for $X$. Because $\mathcal{B}$ is open-finite, we know that Nonempty has a stationary winning strategy $\mathfrak{S}$ in $\mathrm{Ch}(X, \mathcal{B})$, by Theorem 2.16 and Proposition 2.7. Because $\mathcal{B}$ is of countable order, this strategy will also be convergent. We may assume, as in the proof of Proposition 3.7. that $\mathcal{B}=\bigcup_{n<\omega} \mathcal{B}_{n}$, where $\mathcal{B}_{0}=\{X\}$ and each $\mathcal{B}_{n+1}$ is a point-finite refinement of $\left\{\mathfrak{S}(x, U): x \in U \in \mathcal{B}_{n}\right\}$.

The proof now resembles the second part of the proof of Theorem 3.3 . The space $\prod_{n<\omega} \mathcal{B}_{n}$ has a complete metric in which the distance between two distinct sequences is $2^{-n}$ when $n$ is the index of the first position where the sequences differ. Let $S$ be the set of all descending sequences $\left\langle U_{n}\right\rangle_{n<\omega} \in$ $\prod_{n<\omega} \mathcal{B}_{n}$ of open subsets of $X$ such that for every $n<\omega$, there is some $x \in U_{n+1}$ such that $U_{n+1} \subseteq \mathfrak{S}\left(x, U_{n}\right)$. Then $S$ is a closed subset of $\prod_{n<\omega} \mathcal{B}_{n}$, and thus $S$ is a complete metric space.

Now let $f: S \rightarrow X$ be the unique map such that $\left\{f\left(\left\langle U_{n}\right\rangle_{n<\omega}\right)\right\}=$ $\bigcap_{n<\omega} U_{n}$. It is easy to check that this is an open continuous mapping from $S$ onto $X$, using the same technique as the proof of Theorem 3.3 . To see that $f$ is a compact mapping, note that $f^{-1}(x)$ is a closed subset of $S_{x}$, where

$$
S_{x}=\prod_{n<\omega}\left\{U \in \mathcal{B}_{n}: x \in U\right\} .
$$

Because each $\mathcal{B}_{n}$ is point-finite, $S_{x}$ is homeomorphic to a product of finite discrete spaces and is thus compact.

\section{References}

[Ale60] P. Aleksandrov, On the metrization of topological spaces, Bull. Acad. Polon. Sci. Sér. Sci. Math. Astronom Phys. 8 (1960), 135-140 (in Russian).

[Arh62] A. V. Arhangel'skiı̌, On mappings of metric spaces, Dokl. Akad. Nauk SSSR 145 (1962), 245-247 (in Russian); English transl.: Soviet Math. Dokl. 3 (1962), 953-956. 
[Arh63] A. V. Arhangel'skiǔ, Some metrization theorems, Uspekhi Mat. Nauk 18 (1963), no. 5 (113), 139-145 (in Russian).

[BLR08] H. R. Bennett, D. J. Lutzer and G. M. Reed, Domain representability and the Choquet game in Moore and BCO-spaces, Topology Appl. 155 (2008), 445-458.

[Cho69] G. Choquet, Lectures on Analysis. Vol. I. Integration and Topological Vector Spaces, W. A. Benjamin, New York, 1969.

[GT86] F. Galvin and R. Telgársky, Stationary strategies in topological games, Topology Appl. 22 (1986), 51-69.

[Kec95] A. S. Kechris, Classical Descriptive Set Theory, Grad. Texts in Math. 156, Springer, New York, 1995.

[LN76] W. F. Lindgren and P. J. Nyikos, Spaces with bases satisfying certain order and intersection properties, Pacific J. Math. 66 (1976), 455-476.

[Mar03] K. Martin, Topological games in domain theory, Topology Appl. 129 (2003), 177-186.

[MS08] C. Mummert and F. Stephan, Topological aspects of poset spaces, Michigan Math. J. 59 (2010), 3-24.

[Per76] S. A. Peregudov, Certain properties of families of open sets and coverings, Vestnik Moskov. Univ. Ser. I Mat. Mekh. 31 (1976), no. 3, 25-33 (in Russian); English transl.: Moscow Univ. Math. Bull. 31 (1976), no. 3-4, 19-25.

[Pon60] V. Ponomarev, Axioms of countability and continuous mappings, Bull. Acad. Polon. Sci. Sér. Sci. Math. Astronom. Phys. 8 (1960), 127-134 (in Russian).

[SS78] L. A. Steen and J. A. Seebach, Jr., Counterexamples in Topology, 2nd ed., Springer, New York, 1978.

[Tod84] S. Todorčević, Trees and linearly ordered sets, in: Handbook of Set-Theoretic Topology, North-Holland, Amsterdam, 1984, 235-293.

François G. Dorais

Department of Mathematics

University of Michigan

530 Church Street

Ann Arbor, MI 48109, U.S.A.

E-mail: dorais@umich.edu
Carl Mummert Department of Mathematics Marshall University 1 John Marshall Drive Huntington, WV 25755, U.S.A. E-mail: mummertc@marshall.edu

Received 4 June 2009;

in revised form 4 February 2010 